\title{
Linné et la France : entre botanique et politique
}

\section{Anna Svenbro}

\section{(2) OpenEdition}

Journals

Édition électronique

URL : https://journals.openedition.org/rbnu/1961

DOI : 10.4000/rbnu. 1961

ISSN : 2679-6104

\section{Éditeur}

Bibliothèque nationale et universitaire de Strasbourg

\section{Édition imprimée}

Date de publication : 1 novembre 2013

Pagination : 26-37

ISBN : 9782859230517

ISSN : 2109-2761

\section{Référence électronique}

Anna Svenbro, «Linné et la France : entre botanique et politique », La Revue de la BNU [En ligne], 8 | 2013, mis en ligne le 01 novembre 2013, consulté le 18 mai 2021. URL : http:// journals.openedition.org/rbnu/1961 ; DOI : https://doi.org/10.4000/rbnu.1961

\section{(c) $\odot \Theta(\odot)$}

La Revue de la BNU est mise à disposition selon les termes de la Licence Creative Commons Attribution - Pas d'Utilisation Commerciale - Partage dans les Mêmes Conditions 4.0 International. 

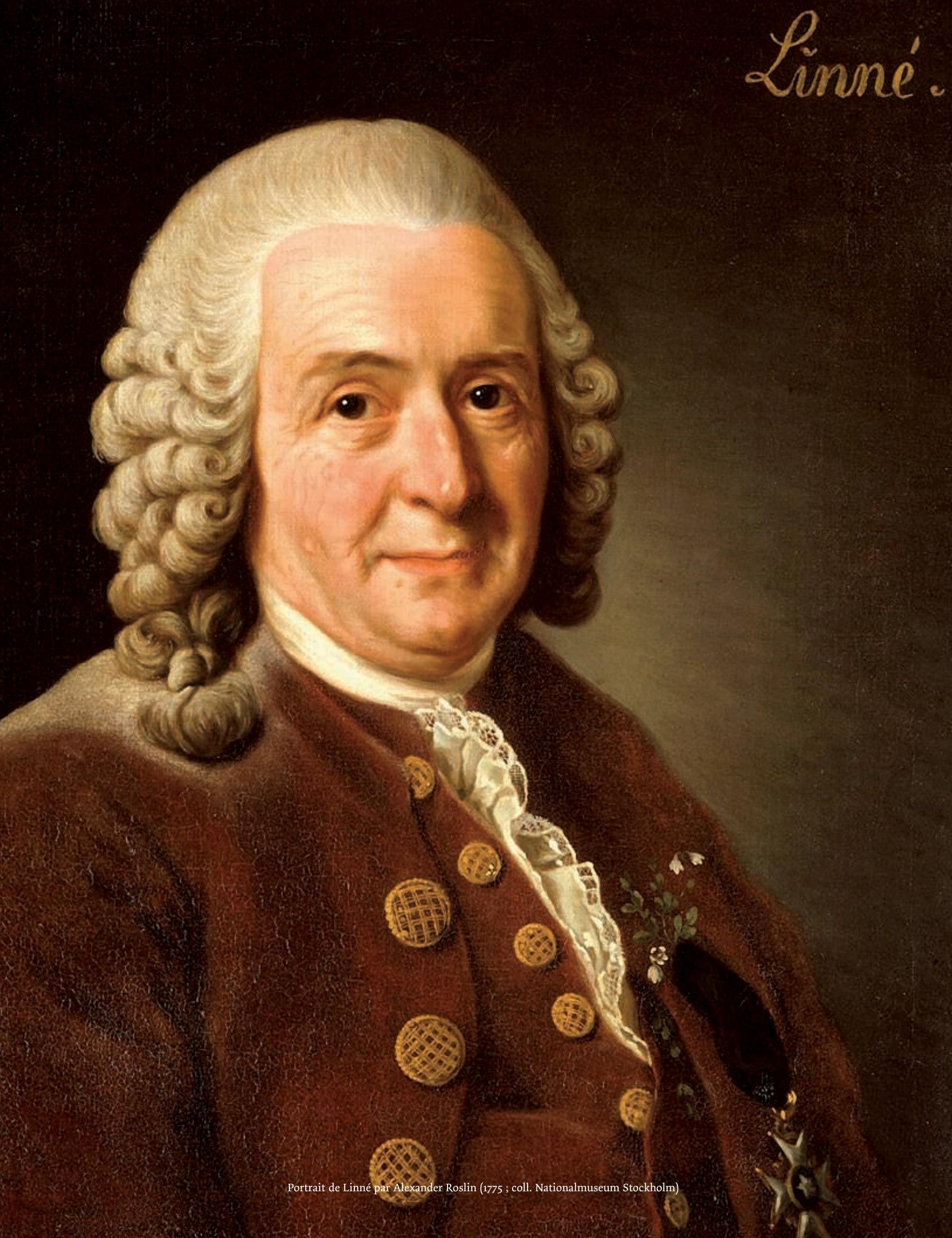


\section{Linné et la France : entre botanique et politique}

$\mathrm{F}$

aire une courte synthèse des relations très complexes que Carl von Linné (1707-1778) ${ }^{1}$ d'une part, et son héritage d'autre part, entretiennent avec la France et la communauté scientifique française du $18^{e}$ et du début du $19^{\mathrm{e}}$ siècles, à partir d'une énorme bibliographie linnéenne qui compte plusieurs milliers de références (analysées notamment par Pascal Duris dans sa thèse de doctorat) est un exercice pour le moins difficile. Or les relations avec la France, pour ce qui concerne l'histoire des sciences, sont capitales pour comprendre la manière dont celles du "Pline du Nord " se sont répandues en Europe, et dont Linné en est venu à constituer une figure capitale dans l'histoire de la botanique, de la zoologie, de la biologie, voire de l'écologie.

La France est de prime abord une terre d'opposition et de controverses pour Linné, particulièrement avec la figure du comte de Buffon, né la même année que lui et mort dix ans plus tard (1707-1788). La France est l'un des pays où l'on débat le plus des théories linnéennes, où les sociétés linnéennes font florès, en relation avec le développement du linnéisme en Angleterre. Car la France ne compte pas que des détracteurs du personnage, elle est aussi le théâtre du développement d'un courant linnéiste très important dont il conviendra de résumer les caractéristiques, qui varient au cours du temps et vont jusqu'à l'idolâtrie.

\section{9-1778 : Systema naturae et Histoire naturelle : Buffon, principal adversaire français de Linné}

Le processus de réception des premières éditions du Systema naturae en France

Pour comprendre ce qui est en jeu dans le caractère d'abord difficile de la réception des idées linnéennes en France, commençons par quelques faits et quelques dates. Le Systema naturce per regna tria naturce, systematice proposita per classes, ordines, genera, et species (Système de la nature, en trois règnes de la nature, divisés en classes, ordres, genres et espèces) paraît pour la première fois à Leyde en 1735, et fait onze pages. Son système compte 24 classes de végétaux, 6 classes d'animaux. Les éditions seront régulièrement revues et augmentées (2 524 pages pour les trois tomes de la douzième édition de 1768, plus de 3000 pages pour la treizième édition de 1770). C'est l'ouvrage majeur de Linné, où il fait son premier essai de hiérarchisation des espèces et de classification systématique des trois règnes de la nature (minéral, végétal, animal) : c'est une révolution dans l'histoire naturelle. Pour la première fois, les connaissances en botanique, zoologie et géologie sont pensées en étant classées, ordonnées suivant une systématicité rigoureuse.

Ce projet, à la base des notions de nomenclature (comment nommer un genre, puis une espèce) et de taxinomie (comment les classer) dans la pensée scientifique moderne, trouve son expression aboutie à partir de la dixième édition de 1758, où son système de nomenclature binomiale des espèces, déjà systématisé pour les plantes dans le Species plantarum de 1753, est généralisé. 


\section{LINNÉ}

C'est par cet ouvrage, publié en latin (langue scientifique de l'époque) que Linné sera d'abord connu en France, et c'est contre les théories développées dans cet ouvrage que vont se cristalliser les oppositions françaises, et en particulier celle de Buffon.

La première salve d'attaques publiques est émise en 1749, dans le premier discours de l'Histoire naturelle, générale et particulière, avec la description du Cabinet du roi, de Buffon, qui porte comme sous-titre De la manière d'étudier et de traiter l'Histoire naturelle. Buffon avait déjà fait état de critiques dans sa correspondance privée en 1745, où la méthode de Linné était décrite au physicien genevois Jalabert comme « la moins sensée et la plus monstrueuse de toutes ". Dans cette attaque directe, la méthode linnéenne est vue par Buffon comme confondant "les objets les plus différents, comme les arbres avec les herbes, [mettant] ensemble \& dans les mêmes classes le mûrier et l'ortie, la tulipe \& l'épine-vinette, l'orme \& la carotte ". En outre, si l'on ne peut voir les différences, notamment, selon Buffon, au niveau des étamines, " on ne sait rien, on n'a rien vu ». Pour lui, les six classes zoologiques sont imparfaites : " il faut bien avoir la manie de faire des classes pour mettre ensemble des êtres aussi différents que l'homme \& le paresseux, ou le singe \& le lézard écailleux ". Il conclut : " Ne serait-il pas plus simple, plus naturel et plus vrai de dire qu'un âne est un âne, \& un chat un chat, que de vouloir, sans savoir pourquoi, qu'un âne soit un cheval, et un chat un loupcervier?"

\section{Les principaux éléments de la controverse avec Buffon et quelques autres}

On peut résumer, comme le fait Giulio Barsanti, l'opposition entre Linné et Buffon à partir d'un certain nombre de critères :

\begin{tabular}{|c|c|}
\hline Caractérisation & Objection ou exception \\
\hline $\begin{array}{l}\text { Logicien (aristotélicien } \\
\text { scolastique) }\end{array}$ & $\begin{array}{l}\text { Grand observateur, } \\
\text { sa formation n'insiste } \\
\text { pas sur la logique. }\end{array}$ \\
\hline Rationaliste, scientifique & $\begin{array}{l}\text { Linné croit en la } \\
\text { numérologie. Son rationa- } \\
\text { lisme est « mystique ». } \\
\text { Il est superstitieux. }\end{array}$ \\
\hline $\begin{array}{l}\text { Discontinuité } \\
\text { des groupes }\end{array}$ & $\begin{array}{l}\text { Insère l'homme parmi les } \\
\text { animaux. « La nature ne fait } \\
\text { pas de saut ». Les groupes } \\
\text { sont comme des territoires } \\
\text { sur une carte (Philosophie } \\
\text { botanique, paragraphe 77). }\end{array}$ \\
\hline Ordre & $\begin{array}{l}\text { Linné remanie beaucoup } \\
\text { ses travaux d'une édition } \\
\text { à l'autre, change les noms... }\end{array}$ \\
\hline $\begin{array}{l}\text { Réaliste : le genre et l'espèce } \\
\text { sont des essences. Dieu } \\
\text { a inscrit dans les choses } \\
\text { les « marques » réelles } \\
\text { par lesquelles nous les } \\
\text { connaissons et classons. }\end{array}$ & $\begin{array}{l}\text { Ordres et classes sont en } \\
\text { partie des œuvres d'art. } \\
\text { Les variétés sont les produits } \\
\text { de la culture. }\end{array}$ \\
\hline $\begin{array}{l}\text { Ordre par hiérarchisation } \\
\text { des caractères : on peut ne } \\
\text { se servir que de quelques } \\
\text { parties (plus pertinentes } \\
\text { que d'autres). }\end{array}$ & $\begin{array}{l}\text { Linné recommande de } \\
\text { consulter l'habitus en secret } \\
\text { et de ne pas tenir trop } \\
\text { fermement aux marques } \\
\text { (« notae »). }\end{array}$ \\
\hline $\begin{array}{l}\text { Classification (création } \\
\text { de macro-ensembles) }\end{array}$ & \\
\hline Donne un tableau général & \\
\hline $\begin{array}{l}\text { Nature économe. } \\
\text { Nature rationnelle. } \\
\text { Providentialisme. } \\
\text { La nature exprime Dieu. }\end{array}$ & \\
\hline $\begin{array}{l}\text { Connaissance de l'espèce } \\
\text { par définition }\end{array}$ & \\
\hline
\end{tabular}


BUFFON

\begin{tabular}{l|l}
\hline \multicolumn{1}{c|}{ Caractérisation } & \multicolumn{1}{c}{ Objection ou exception } \\
\hline $\begin{array}{l}\text { Empiriste (observation, } \\
\text { description). " Observation des échantillons de } \\
\text { tout ce qui peuple l'Univers », " voir beaucoup, } \\
\text { revoir souvent ». }\end{array}$ & $\begin{array}{l}\text { Auteur d'hypothèses et de grandes « vues » sur la } \\
\text { nature. Dépasse l'histoire (collection de faits) par } \\
\text { la philosophie (système général). }\end{array}$ \\
\hline
\end{tabular}

Philosophe, esprit libre

La distinction entre science et philosophie ne vaut pas au $18^{\mathrm{e}}$ siècle, où la philosophie est le corps complet du savoir.

Continuiste : affirme que la nature va par nuances et que " tout ce qui peut être, est ". On passe "par degrés presqu'insensibles " d'une espèce à une autre. "Il se trouve un grand nombre d'espèces moyennes et d'objets mi-partis qu'on ne sait où placer et qui dérangent nécessairement le projet du système général ».

Désordre

Discontinuité radicale de l'homme.

Séparation entre les espèces (claire pour l'âne et le cheval).

Buffon recherche des lois générales, et croit que par comparaison, on peut connaître véritablement la nature.

Nominaliste : il n'existe que des individus. Les noms des catégories générales (genres ou familles) sont des conventions.

Réalité de l'espèce. Buffon recourt à des genres et à des familles.

Invite à ne pas juger par une seule partie mais à partir du « tout ensemble ».

Buffon ne vise pas du tout une classification (pour lui, il s'agit d'une science abstraite, comme les mathématiques).

Spécification (isoler les espèces qui sont les vrais êtres de la nature)

Donne une vraie physique qui décrit le réel (et non mathématique ou systématique, disciplines abstraites)

Nature aveugle. Nature qui dépasse nos capacités. Épicurisme. Dieu sans rapport avec la nature.

Connaissance de l'espèce par description : se résume très bien sur une gravure ou une planche. 


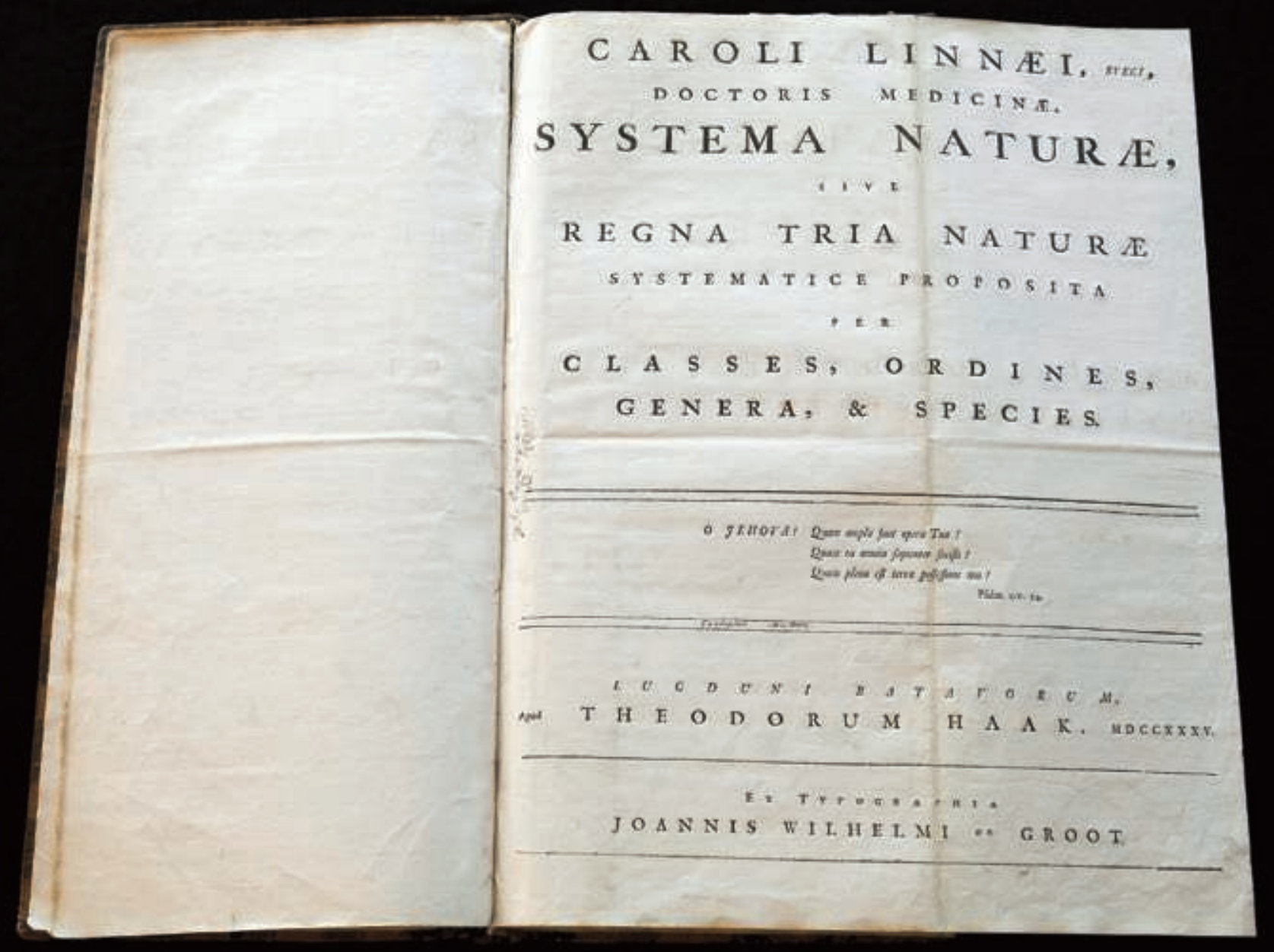


D'autres naturalistes et des philosophes français critiqueront la systématique linnéenne, notamment Julien Offray de La Mettrie, Diderot, Maupertuis (qui d'ailleurs avait entrepris, comme Linné mais pour des raisons différentes - mesurer la longueur d'un arc polaire et d'un arc équatorial pour déterminer la forme de la Terre - un voyage en Laponie), Adanson, Lamarck, Antoine-Laurent de Jussieu (qui met au point avec ses Genera plantarum un autre système de classification " par affinités naturelles "). Les principaux reproches autour des théories linnéennes ont trait à leur caractère artificiel et fixiste. L'entreprise de Linné ne fait que partiellement appel à la raison, et il y a peu d'incitation à l'expérimentation. Les savants français lui reprochent aussi une démarche empreinte de religiosité.

\section{La difficile première implantation du linnéisme en France}

Difficile pour Linné, a priori, dans un pays où l'intendance du Jardin du roi (actuel Jardin des plantes à Paris) est confiée à Buffon en 1739, de se faire une place. L'élite scientifique parisienne restera majoritairement opposée au système de Linné et à sa nomenclature binomiale jusqu'à la Révolution. Linné trouvera un accueil beaucoup plus favorable en province, si l'on en croit sa correspondance, avec quelques fiefs précoces : Montpellier, Lyon, sur lesquels on s'attardera un peu, mais aussi Bordeaux, Agen, Toulouse, Strasbourg et Nancy.

Montpellier a aussi, depuis 1593, un Jardin des plantes, et un médecin-botaniste influent, François Boissier de La Croix de Sauvages (1706-1767), qui va à Paris puis à Leyde dans les années 1730, y croise Boerhaave, connaissance commune avec Linné, et qui va appliquer une méthode botanique de classement des maladies dans sa Nosologia methodica, où l'on trouve dix classes de maladies, 295 genres et 2400 espèces. Il ne rencontre jamais Linné, mais échange avec lui une correspondance fournie de 1737 à 1765 . Grâce à lui, Linné est nommé associé étranger à la Société royale des sciences de Montpellier en 1743, Boissier étant élu à l'Académie royale des sciences de Stockholm en 1749. En 1751, Boissier fait paraître le Methodus foliorum (méthode pour reconnaître les plantes par les feuilles), directement inspiré des idées linnéennes (classification pratique, nomenclature fixe).

Autre Montpelliérain capital dans la réception de Linné, Antoine Gouan (1733-1821), qui se met en rapport avec lui à l'instigation de Boissier. En 1762, il publie un
Hortus regius Monspeliensis qui est un catalogue du jardin botanique de Montpellier établi selon le système linnéen. La Flora Monspeliaca suit trois ans plus tard. Il est le premier à avoir l'idée de fonder une société linnéenne en France, projet qui n'aboutira pas à Montpellier avant 1820.

À Lyon, berceau de la famille Jussieu, on apprécie aussi beaucoup Linné et ses idées, grâce à trois hommes, Charles-Joseph Devillers (1724-1810), Marc-Antoine-Louis Claret de Felrieu de la Tourette (1729-1793) et JeanEmmanuel Gilibert (1741-1814). Devillers, mathématicien et physicien à l'origine, entré à l'Académie de Lyon en 1763 , possède un riche cabinet d'antiques et de curiosités. Or il va herboriser avec La Tourette et Gilibert en Suisse et publier la partie entomologique de l'œuvre de Linné sous le nom de Caroli Linnaei entomologia juste avant la Révolution. La Tourette, lui, est un ami de JeanJacques Rousseau. Il entretient une correspondance tant avec Linné qu'avec Jussieu. Il publie en 1766 ses Démonstrations élémentaires de botanique qui suivent les méthodes de Linné en les combinant habilement avec celles de Tournefort, qu'on range plutôt dans le camp de Buffon. Son Voyage au mont Pilat suit aussi la méthode linnéenne. Gilibert enfin, médecin et naturaliste, l'un des fondateurs de l'École centrale de Lyon, voyage beaucoup en Europe, en Pologne notamment, jusqu'à son retour à Lyon en 1783. Il est élève de Boissier, cité précédemment. Son séjour en Pologne lui donne l'occasion de publier une Flora Lithuanica, et il fait paraître en France les meilleures thèses de botanique soutenues sous la présidence de Linné. Enfin, on peut citer à Strasbourg Jean Hermann et Pierre-Rémi Willemet.

\section{8-1790 : la réception de Linné dans les dernières années de l'Ancien Régime et le règne de Buffon}

\section{Le succès des idées linnéennes à Paris passe par la Grande-Bretagne}

À Paris, il faudra attendre l'impulsion du Britannique James Edward Smith pour que les idées de Linné puissent susciter le débat dans les communautés de savants. La botanique britannique de la seconde moitié du $18^{\text {e }}$ siècle sera bien influencée par Linné, avec l'Irlandais Patrick Browne (1720-179o), médecin lui aussi, mais surtout avec William Hudson (1730-1793) et sa Flora anglica qui suit totalement les préceptes du linnéisme avec la nomenclature binomiale des espèces, et enfin avec Joseph Banks (1743-1820), l'un des compagnons de voyage de Cook en 
1769 (ce voyage lui permettra de rapporter des échantillons de flore et de faune remarquables en Angleterre). Banks présidera la Royal Society de 1778, l'année de la mort de Linné, à sa propre mort en 1820 . Les jardins de Kew Gardens sont arrangés selon le système linnéen, et seront sous la responsabilité de Banks jusqu'à cette dernière date.

Mais Smith sera déterminant pour le cas français qui nous préoccupe. Il se porte en effet acquéreur en 1783 des 3 ooo ouvrages, 5 caisses de plantes, 4 caisses de minéraux, 2 caisses d'insectes, et des 36 caisses de lettres et papiers divers des collections de la bibliothèque de Linné. Smith est médecin, il soutient son doctorat à Leyde en 1786 , et va entamer après cela ce que l'élite cultivée appelle le "Grand Tour » (Hollande, France, Suisse, Italie). Il séjourne à Paris de fin juillet à fin octobre 1786 ; il y est guidé dans la ville par Broussonnet. Les deux hommes vont faire plusieurs rencontres marquantes, et notamment celle de Joseph Dombey, de retour d'Amérique du Sud. Dombey est rattaché à l'école montpelliéraine, et a vanté lors de son séjour au Chili la classification linnéenne. Smith et Broussonnet séjournent aussi chez Louis de Noailles, ardent défenseur du linnéisme, qui a correspondu à partir de 1752 avec le savant suédois. Smith rencontre également Thouin, jardinier en chef au Jardin du roi, qui avait correspondu avec Linné de 1770 à sa mort, et lui montre ses collections et son herbier personnels. Smith va donc consolider ses relations avec divers naturalistes français plus ou moins familiers ou partisans de la méthode linnéenne, et son influence sera capitale : le 28 décembre 1787 est fondée, deux mois avant celle de Londres, la première Société linnéenne de Paris, la première au monde.

\section{La fondation de la première Société linnéenne de Paris et ses conséquences}

Un manuscrit en latin conservé à la Bibliothèque Mazarine (portant le numéro 4441) permet de faire l'histoire des procès-verbaux de cette première société, dont les membres se sont réunis, 42 fois en tout, du 28 décembre 1787 au 26 décembre 1788. Les séances sont hebdomadaires jusqu'au 18 septembre 1788 , mensuelles ensuite, pour redevenir hebdomadaires le dernier mois d'existence de la société. On y croise les linnéens fidèles dont on détaillera le parcours un peu plus tard, mais aussi des opposants, comme La Mettrie, des naturalistes étrangers, comme le Suédois Anders Erikson Sparrman. Faute d'organe de diffusion, on expose ses travaux au cours des réunions avant de les publier dans des revues comme le Journal de physique ou le Journal des savants. Broussonnet donne lecture de sa traduction, à partir du texte anglais établi par Smith, de la dissertation de Linné Sur les sexes des plantes. On y présente des ouvrages français ou étrangers d'histoire naturelle. On part en excursion pour herboriser aux environs de Paris, lors d'une " fête linnéenne ", le jour de l'anniversaire du savant, le 24 mai 1788. Cette première société linnéenne disparaît du fait du climat politique tendu en France (on est à la veille des états généraux, qui ont déjà été convoqués), et aussi peut-être (même si cette hypothèse doit être énoncée avec précaution étant donné qu'elle a été avancée par les linnéens) du fait des adversaires de Linné à Paris.

\section{Quelques linnéens remarquables}

Les membres fondateurs de la première Société linnéenne de Paris sont au nombre de cinq. Louis-AugustinGuillaume Bosc d'Antic (1759-1828), futur professeur de culture au Jardin des plantes et à l'École vétérinaire d'Alfort, est très tôt attiré par le système de Linné, ses méthodes rigoureuses et son langage concis et précis. Il publie de nombreux mémoires dans divers journaux scientifiques de l'époque, et il est l'un des rédacteurs du Nouveau dictionnaire d'histoire naturelle de Déterville et du Dictionnaire d'agriculture.

Pierre-Marie-Auguste Broussonnet (1761-1807) est né à Montpellier où il est l'élève de Gouan. Il est médecin à dix-huit ans, membre à vingt-deux ans de la Royal Society de Londres et à vingt-quatre ans de l'Académie royale des sciences de Paris. Il va se lier d'amitié avec un grand nombre de naturalistes européens plus ou moins engagés dans la propagation du linnéisme sur le continent, dont Sparrman, Banks et Smith. Il est nommé en 1784 suppléant au Collège royal (actuel Collège de France), professeur adjoint d'économie rurale à l'École vétérinaire d'Alfort, et l'année suivante secrétaire de la Société royale d'agriculture de Paris. Comment un défenseur de Linné a-t-il pu faire carrière à Paris ? Certes, une telle carrière n'est pas commune, mais on peut cependant l'expliquer. Buffon, Jussieu et leurs partisans dominent la scène. Broussonnet, lui, se présente d'abord comme voulant effectuer la synthèse entre la vision buffonienne philosophique de la nature et l'exactitude et la précision des méthodes linnéennes. Son Ichtyologie de 1782 applique pour la première fois la nomenclature linnéenne à la zoologie en France. C'est la méthode de Linné, et non son système de classification, qui compte pour lui. 


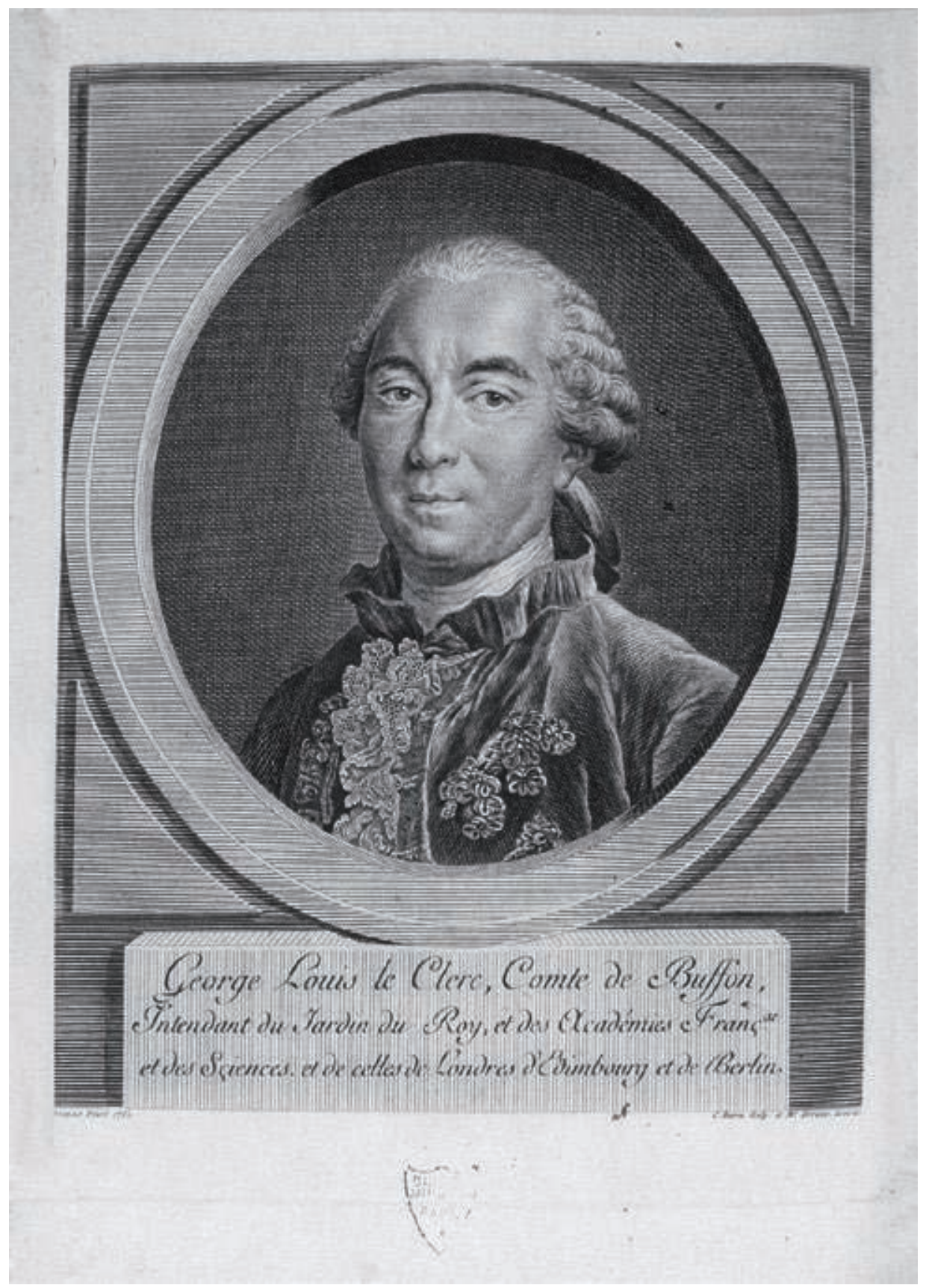

Portrait de Buffon (frontispice de l'édition de 1774 de l'Histoire naturelle, gravé par Baron sur un dessin de Drouais ; coll. BnF-Gallica) 


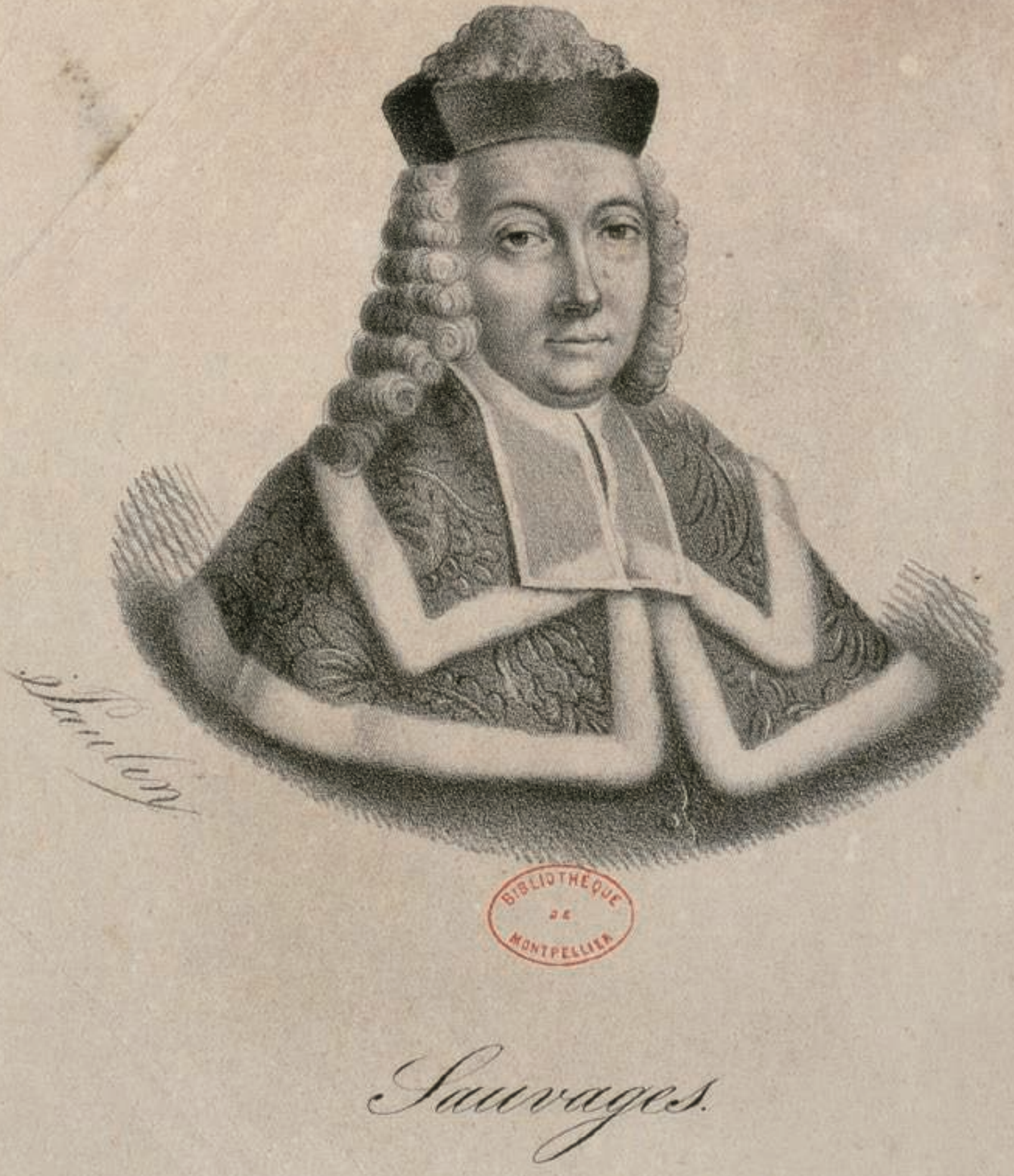

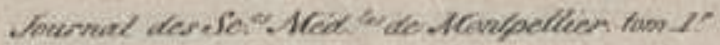

Lathite didagmin or:

Portrait de Boissier de La Croix de Sauvages (lithographie d'Edmond-Ernest Paulin, $19^{\mathrm{e}}$ siècle ; coll. Bibliothèque municipale de Montpellier) 
Aubin-Louis Millin de Grandmaison (1759-1818) est un polygraphe, un homme de lettres, un traducteur, qui publie des Mélanges de littérature étrangère où se trouve la traduction en français de l'Éloge de Linné d'Abraham Bäck. Il traduira en 1789 la Revue générale des écrits de Linné de Pulteney. Pendant son emprisonnement sous la Terreur en 1794, il rédige des Éléments d'histoire naturelle. La Terreur finie, il est nommé conservateur du cabinet des médailles et antiques de la Bibliothèque nationale, et il fonde avec Noël et de Warens Le Magasin encyclopédique, appelé à succéder au Journal des savants. Il a une connaissance surtout livresque de Linné, et il est très rapidement informé, du fait de sa connaissance des langues, des divers courants de pensée européens. Mais même lorsqu'il se consacrera à la numismatique et aux antiquités, il reprendra les méthodes linnéennes en élaborant un système de classification des monnaies grecques inspiré des méthodes du Suédois.

Guillaume-Antoine Ollivier (1756-1814), quant à lui, fait ses études médicales à Montpellier où il est disciple de Gouan et condisciple de Broussonnet, qui lui présente d'ailleurs Smith. Il entreprend la rédaction d'une Entomologie ou Histoire naturelle des Insectes. Il sera comme Bosc d'Antic professeur à l'École vétérinaire d'Alfort. Enfin, André Thouin (1747-1824) est le jardinier en chef du Jardin du roi, et dépend de Buffon qui en est l'intendant. Il devient membre en 1784 de la Société royale d'agriculture et entre en 1786 à l'Académie royale des sciences. C'est lui qui va dresser (avec Desfontaines) l'inventaire des collections botaniques (cabinets, jardins) des émigrés pour compléter les collections du Muséum. Subordonné de Buffon, cela ne l'empêchera nullement de correspondre avec Linné de 1770 à 1778.

Mais mis à part Thouin, tous sont très jeunes, et sont animés par les nouvelles idées politiques. Ils accueillent tous avec ferveur la Révolution, à laquelle ils participent activement. Ils défendent une idée républicaine et modérée de la Révolution, ce qui ne sera pas sans conséquences, notamment sous la Terreur.

\section{0-1830 : le linnéisme à la française, un naturalisme révolutionnaire?}

\section{Linné, figure de la science révolutionnaire contre Buffon représentant l'Ancien Régime}

La Révolution française met Linné et son système à la mode, renforçant son impact en France. D'une part, Buffon est trop associé à l'Ancien Régime pour ne pas paraître suspect, même s'il continuera d'avoir bon nombre de partisans au cours de la période. Mais Linné a le vent en poupe pendant la Révolution, et le doit en partie à Rousseau, qui s'est intéressé à partir de 1762 à la botanique, lui accordant un statut de science à part entière. Les premiers ouvrages de botanique dont Rousseau prend possession sont ceux de Linné. "Seul avec la nature et vous, je passe dans mes promenades champêtres des heures délicieuses, et je tire un profit plus réel de votre Philosophia botanica que de tous les livres de morale ", écrit Rousseau à Linné en 1771. Dans ses Fragments pour un dictionnaire des termes d'usage en botanique, rédigés en 1774 et publiés en 1781 , Rousseau est un ardent défenseur du savant. Selon lui, Linné « a un peu tiré la botanique des écoles de pharmacie pour la rendre à l'histoire naturelle et aux usages économiques ". Mais le rapport de Rousseau à la nomenclature est ambigu ; c'est un " savoir herboriste " qu'il trouve parfois pédant. Pourtant, il se demande " comment il est possible de s'attacher à l'étude des plantes, en rejetant celle de la nomenclature ", linnéenne bien sûr. Rousseau devenant pour la Révolution une figure politique tutélaire, le contenu botanique de son œuvre intéresse, et ce sont des philosophes botanistes qu'on honore lors de fêtes. En effet, les révolutionnaires vont mettre en pratique le culte de la nature, de l'éducation, l'amour du végétal faisant partie intégrante de cette éducation. L'heure est à la botanique citoyenne : on plante des arbres de la liberté, qu'il faut choisir avec soin. Le calendrier républicain devient champêtre. Les sciences naturelles sont enseignées dans les Écoles centrales qui ont remplacé les collèges de l'Ancien Régime.

Le 24 messidor an VI, le ministre de l'Intérieur indique par lettre aux professeurs d'histoire naturelle des Écoles centrales qu'il doivent de préférence distribuer comme récompenses en fin d'année ou dans les fêtes nationales " les discours de Buffon, ses Époques de la Nature, Réaumur, Bonnet, Tournefort, Jussieu, et surtout Linné ". Les traducteurs, Millin en tête, vantent le laconisme révolutionnaire pétri de culture antique des œuvres du Suédois. Le système des nomenclatures sera étendu à la chimie avec Lavoisier, à l'anatomie avec Vicq d'Azyr et Dumas, à la minéralogie, à la numismatique par Millin. On érige en 1790 un buste à Linné dans le Jardin des plantes, dont Buffon avait été l'intendant (il disparaîtra un temps sous la Terreur). Il y aura même une controverse sur son nom : que choisir, Linné (nom certes francisé, mais nom d'anoblissement du personnage, donné par un roi) ou Linnæus ? Il est décidé en tout cas de ne plus employer la particule nobiliaire par les 
naturalistes qui se réunissent le jour de son anniversaire en 1790. Ces mêmes naturalistes choisissent "Linnæeus, à qui le roi de Suède avait donné le nom de Linné pour l'anoblir, et à qui nous, Français libres, avons rendu celui de Linnous, pour l'honorer davantage ". Cette position se durcit à la chute de la royauté en 1792.

\section{Les fêtes linnéennes et le culte de Linné}

La dette à Linné va prendre la forme d'un véritable culte en France, et ce bien au-delà de la période révolutionnaire. D'une part, les sociétés linnéennes essaiment partout après la Révolution et l'Empire : Bordeaux en 1818 , Paris en 1821 , Lyon en 1822 , Caen en 1823 , etc. Toutes ces sociétés, ouvertes aux femmes, célèbrent un véritable culte de Linné, notamment lors de fêtes savantes champêtres, dites fêtes linnéennes, dont le rituel est soigneusement codifié. D'après la synthèse que fait Pascal Duris, à partir des divers comptes rendus faits à l'époque par ces sociétés, les fêtes ont lieu, généralement, soit le 24 mai, anniversaire de Linné, soit le premier jeudi suivant la Saint-Jean, comme à Bordeaux.

La fête linnéenne est réservée aux membres des sociétés et à leurs proches. À Paris, ils portent un brin de Linncea borealis en signe de reconnaissance. Des personnalités extérieures peuvent néanmoins être invitées. Le nombre des participants aux fêtes linnéennes est très variable. Près d'une centaine à Paris, un peu moins sans doute à Bordeaux, ils ne sont que quelques-uns dans les fêtes organisées par les sections. Il peut même arriver qu'une ou deux personnes seulement rendent hommage au savant.

La fête linnéenne commence très tôt : dès 3 ou 4 heures du matin à Libourne, Narbonne ou Montpellier, afin d'éviter les grosses chaleurs de la journée, vers 5 ou 6 heures à Bordeaux, entre 7 et 9 heures à Paris. Les linnéens gagnent le lieu de la fête par petits groupes et mettent à profit le trajet pour herboriser. Si l'excursion a commencé tôt, un déjeuner "frugal " est servi vers 10 heures. C'est l'occasion de porter une première fois des toasts à la mémoire de l'« immortel Linné ». Puis l'herborisation se poursuit jusqu'à midi.

À midi exactement, heure à laquelle on pensait que Linné était né, tous les participants se retrouvent sur le lieu de la fête pour effectuer des relevés météorologiques : l'aspect du ciel, la force et la direction du vent, les météores éventuels, la température, l'hygrométrie et la pression barométrique sont scrupuleusement consignés dans le procès-verbal de la journée. La simultanéité de ces mesures partout en France et dans le monde est une obsession. La section de l'île Maurice de la Société linnéenne de Bordeaux décide ainsi en 1823 que la fête " commencera à quatre heures de l'après dînée, afin de mettre le plus d'unité possible avec les sociétés qui doivent la célébrer en Europe. En effet, Bordeaux, la métropole des sections linnéennes, se trouvant à peu près à 58 degrés de longitude occidentale, il en résulte que l'heure de la séance commençant à midi, il doit être près de 4 heures à Maurice, en comptant 4 minutes de différence par degrés, d'après le calcul des astronomes consigné dans la connaissance des temps ".

Vers midi trente, le président ouvre la séance qui se tient parfois dans des lieux insolites. Dans une lettre datée de 1824 à Jean-François Laterrade, botaniste linnéen bordelais, le voyageur et naturaliste René-Primevère Lesson raconte comment, alors qu'il participe avec Dumont d'Urville et le médecin ornithologiste Prosper Garnot à une expédition scientifique autour du monde à bord du navire La Coquille, il porte avec ceux-ci un toast à Linné lors d'une escale aux Malouines le 4 décembre 1822 : "Nous serons sans contredit, écrit Lesson, les premiers Européens qui l'auront porté par 52 degrés de lat. S. et qui auront élevé un concert de louanges aux mânes du grand Linné, sur ces îles désertes, habitations des phoques et des pingouins, mais célèbres par les herborisations du savant Commerson ".

En 1823, deux correspondants de la Société linnéenne de Bordeaux célèbrent la fête linnéenne dans une maison de campagne du roi Radama, à Madagascar. La fête de la section des Indes se tient en 1824 sur le sommet, battu par les vents, de la montagne du Pouce dans l'île Maurice, et en 1825 dans le tronc creux d'un baobab. À Ogenne, la fête de 1824 se déroule sur un ancien tumulus que la section des Basses-Pyrénées se propose de faire fouiller. Le plus souvent, néanmoins, la séance a lieu dans le parc d'un château, à l'ombre d'arbres séculaires, ou dans une clairière. Les bois de Meudon, de Vincennes, de Romainville, de Saint-Cloud, de Ville-d'Avray offrent, avec la forêt de Saint-Germain, le village de Fontenay-aux-Roses, les îles de la Marne et le parc de Sceaux, d'excellents sites très prisés des linnéens parisiens.

Le site de la fête linnéenne est décoré avec soin. Le portrait de Linné, ceint d'une couronne emblématique de vingt-quatre fleurs illustrant chacune des classes du système sexuel, est placé au-dessus du fauteuil du président. 
Pour conclure, on citera quelques vers écrits en 1808 par Jacques Delille :

"Linné, réjouis-toi : le Nord vit ta naissance, Mais ton plus beau trophée enorgueillit la France. Elle ne choisit point pour y placer tes traits, Ou l'ombre d'un lycée, ou les murs d'un palais ; Mais dans ce beau jardin où l'enceinte féconde Accorde une patrie à tous les plans du monde [...] Viens : dans cet Élysée, autrefois son domaine, L'ombre du grand Buffon attend déjà la tienne ; Et de tous les climats, et de toutes saisons, Les fleurs briguent l'honneur de couronner vos fronts ".

Ces quelques vers suffisent à résumer le prestige, allant jusqu'au culte, dont jouit l'auteur de la nomenclature binaire des végétaux et des animaux, de la période révolutionnaire jusqu'au milieu du $19^{\mathrm{e}}$ siècle.

Mais la parution, en 1859, de L'Origine des espèces de Darwin semble changer la donne du rapport de la France savante à Linné et à son héritage. Linné y est vu comme un tenant de la fixité des espèces, Buffon trouvant en revanche une place de précurseur de la théorie évolutionniste. On l'a vu précédemment, le tableau est beaucoup plus complexe que cette perception commune. La France compte aujourd'hui encore une demi-douzaine de sociétés linnéennes (qui ne sont pas devenues des sociétés d'histoire naturelle comme à Paris, mais qui revendiquent explicitement l'héritage linnéen) encore en activité (à Lyon, dans le Nord-Picardie, à Bordeaux, en Normandie, en Seine-Maritime, en Provence), et dont le but est " d'accélérer les progrès de l'histoire naturelle et principalement la connaissance des richesses des trois règnes ${ }^{2}$ dans leurs régions d'implantation respectives, apportant leur contribution à la vie scientifique et à l'histoire des sciences locales. Et la Commission internationale de nomenclature zoologique de prendre comme point de départ celle qu'a donné Linné en 1758. Ce ne sont pas seulement Jacques Delille et ses contemporains, mais encore les conservateurs du Muséum d'histoire naturelle qui ont choisi de commémorer Linné en compagnie de Buffon, son contradicteur, en 2007. L'histoire des relations entre l'œuvre de Linné et la France est non seulement riche, mais encore loin d'être terminée.

\section{Anna Svenbro}

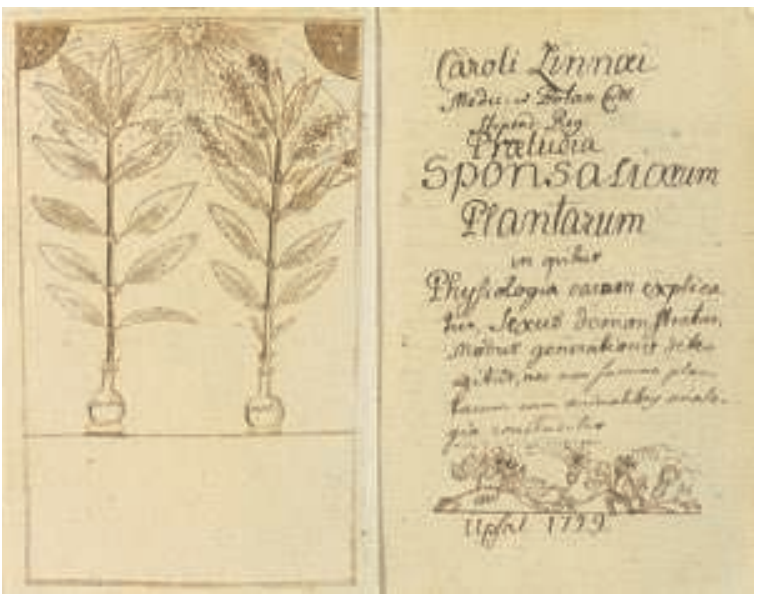

Page de titre du manuscrit de Linné des Praeludia sponsaliorum plantarum (coll. Bibliothèque de l'université d'Uppsala)

\section{Notes}

1 - De son vrai nom Karl (ou Carl) Linnæus

2 - Citation extraite du premier règlement de la Société linnéenne de Lyon, et reprise par Raymond Ramousse, ancien président de la société, dans la présentation du 23 novembre 2010 sur le site internet de celle-ci : http://sll-section-generale.e-monsite.com/pages/histoire/presentationde-la-societe-linneenne-de-lyon-par-r-ramousse/.

\section{ORIENTATIONS BIBLIOGRAPHIQUES :}

Giulio Barsanti / Linné et Buffon : deux visions différentes de la nature et de l'histoire naturelle, in Hoquet, Thierry (dir.), Les fondements de la botanique : Linné et la classification des plantes, Paris : Vuibert, 2005

Pietro Corsi / Décrire ou classer ? Taxinomies au XVIII siècle, in Hoquet, Thierry (dir.), Les fondements de la botanique: Linné et la classification des plantes, Paris : Vuibert, 2005

Pascal Duris / Linné et la France (1780-1850), Genève : Droz, 1993

Pascal Duris / Linné révolutionnaire?, in Pour la science, février-mai 2006, pp. 78-89

Pascal Duris | Le culte de Linné, in Pour la science, février-mai 2006, pp. 98-111

Thierry Hoquet / Linné et les guerres des plantes, in Hoquet, Thierry (dir.), Les fondements de la botanique : Linné et la classification des plantes, Paris : Vuibert, 2005 